\title{
Dialogische Interaktion und kognitive Modelle in der neuropsychologischen Rehabilitation
}

\author{
Nergiz Turgut ${ }^{1,2}$ \\ Institute of Neurosciences, Universidad San Francisco de Quito, Ecuador \\ Colegio de Ciencias Sociales y Humanidades, Universidad San Francisco de Quito, Ecuador
}

\section{Einleitung}

Der folgende Kommentar bezieht sich auf die Arbeit von Helmut Hildebrandt (2021) ,Zehn Argumente für dialogische Interaktion und kognitive Modelle als Grundlage neuropsychologischer Rehabilitation". Hildebrandt (2021) vermag es in seinem Text, teils komplizierte Sachverhalte mit anschaulichen und praxisrelevanten Beispielen zu erklären, zentrale Komponenten einer erfolgreichen neuropsychologischen Rehabilitation herauszuarbeiten sowie interessante Denkanstöße zu setzen. Dieser Text ist höchst relevant für klinische Neuropsycholog_innen und -psychologen jeder Rehabilitationsstufe sowie klinische Forscherinnen und Forscher mit Interessenschwerpunkt in Neurorehabilitation.

\section{Unawareness}

Hildebrandt (2021) postuliert im Titel des ersten Punktes auf ,ein Recht auf Unawareness" und eröffnet ein Thema, welches zentral für die neurologische Rehabilitation ist, jedoch selten Beachtung findet: „Unawareness ist (...) Grundzustand der PatientInnen nach einer Hirnschädigung () “. Unawareness im Kontext einer kognitiven Störung ist ein häufig eintretendes Phänomen und nicht ausschließlich bei Betroffenen nach rechtshemisphärischer Läsion aufzufinden. Schon frühe Studien zeigen, dass über $70 \%$ der Betroffenen nach Schlaganfall sich ihrer kognitiven Veränderungen nicht „bewusst“ sind (Anderson \& Tranel, 1989). Patienteninnen und Patienten mit Unawareness haben eine negative Prognostik auf den Rehabilita- tionsverlauf und daher sollte die Unawareness adressiert werden, um die Basis einer motivierten Therapie zu schaffen (Hartman-Maeir et al., 2002; Leung \& Liu, 2011). Haben die Betroffenen jedoch ein "Recht auf Unawareness"? Mir ist die Formulierung nicht ganz schlüssig und ich interpretiere sie dahingehend, dass auch Patienteninnen und Patienten, die sich ihrer kognitiven Störung nicht bewusst sind, eine Rehabilitation erhalten können und die Therapie ihre Realität integrieren sollte, anstatt ihr eine Realität aufzuzwingen.

\section{Kognitive Theorien}

Ein sehr interessanter und für mich neuer Ansatz ist die Simulation einer kognitiven Umwelt als Rehabilitationsstrategie, die Hildebrandt (2021) mit sehr anschaulichen Vergleichen bezüglich sensorischer Defizite erklärt. Wie Hildebrandt erläutert, sind kognitive Theorien essenziell, um eine Simulation kognitiver Umwelten zu ermöglichen und somit neuropsychologische Diagnostik und Rehabilitation zu verbessern. Dabei sollten die Theorien störungsspezifisch entwickelt werden und auch akute Phasen einschließen. Einschränkungen und Erweiterungsvorschläge werden im Kontext der Aphasietherapie genannt. Ergänzend zu erwähnen wäre zudem ein Mangel von neuen kognitiven Theorien im Kontext des visuellen Neglects. Bestehende Theorien erklären nur unzureichend, wie z.B. egozentrische und allozentrische Koordinatensysteme verarbeitet werden, welche die Entwicklung von Therapien zur Behandlung der allozentrischen Symptomatik erschweren. 


\section{Definition des Schweregrades}

Hildebrandt (2021) betont, wie wichtig eine Definition des Schweregrades der Störung für eine erfolgreiche Rehabilitation ist. Dabei seien „Handlungsanweisungen (...), die keine systematische Abstufung von Schweregraden umfassen, (...) im besten Fall unvollständig, im schlechten Fall eine Überbelastung oder Demütigung der Patient_innen“". Wichtig und richtig finde ich die Erwähnung der Problematik von reinen Bottom-up-Verfahren, die unabhängig vom Schweregrad der Störung eingesetzt werden und bis heute keine zufriedenstellende Wirksamkeit zeigen (siehe z. B. Azouvi, Jacquin-Courtois und Luauté [2017] zur Behandlung des Neglects). Stattdessen sollten sie in Kombination mit kognitiven Therapien (Top-down-Methoden) eingesetzt werden (Azouvi et al., 2017; Turgut et al., 2018).

Virtual Reality (VR) ist flexibel gegenüber dem Schweregrad der kognitiven Störung und ideal, um die Leistungsgrenzen der Patientinnen und Patienten zu erfassen. Ein großer Nachteil laut Hildebrandt (2021) ist die fehlende Spezifität der Gestaltung einer individuellen kognitiven Umgebung. Dies trifft meiner Meinung nach heutzutage definitiv zu, jedoch könnte sich bald mit dem schnellen technischen Fortschritt in diesem Bereich die Gestaltung verbessern.

\section{Bedeutung der Interaktion zwischen Therapeutinnen/Therapeuten und Patientinnen/Patienten}

Die Bedeutung der therapeutischen Interaktion wird in Punkt 5 (Therapeutin oder Therapeut als Hinweisreiz) erwähnt. Ich könnte mir jedoch vorstellen, dies als neuen Punkt aufzunehmen. In einer Studie zeigt sich, dass während der Schlaganfallrehabilitation die Betroffenen die Qualität der therapeutischen Allianz als vorrangig empfinden, noch vor Inhalt oder der Dauer der Therapie (Peiris, Taylor \& Shields, 2012). Eine weitere Studie zeigt, insbesondere bei Betroffenen nach einem Schlaganfall, wie wichtig es ist, durch personenzentrierte Therapie den Aufbau einer Beziehung zu erlangen (Bishop, Kayes \& McPherson, 2021). Zusammenfügend würde ich sagen (und dies gilt es noch genauer zu untersuchen), dass eine personenzentrierte Therapie mit Berücksichtigung des Schweregrades der Betroffenen zu einer verbesserten therapeutischen Beziehung und eventuell zu einer höheren Motivation und Compliance während der Therapie führt, auch bei Unawareness der eigenen kognitiven Defizite. Die therapeutische Allianz ist zudem ein weiterer Punkt gegen reine Bottom-up-Methoden, da hier die Interaktion größtenteils wegfällt.

\section{Cueing}

Die Bedeutung des Cueings (das Setzen von Hinweisreizen) wird von Hildebrandt (2021) sehr schön mit Beispielen aus der Gedächtnisrehabilitation, Aphasie- und Neglecttherapie veranschaulicht. Dabei ist ein standardisiertes/ strategisches Vorgehen basierend auf dem Schweregrad der Störung zentral, um das gewünschte Verhalten zu erlangen. Nach stabiler Verbesserung werden die Cues schrittweise reduziert, um die Abhängigkeit zur Therapeutin oder zum Therapeuten zu reduzieren und Selbstständigkeit aufzubauen. Dabei spielt die Arbeitsgedächtnisleistung womöglich eine zentrale Rolle.

\section{Frühe Intervention}

Hildebrandt (2021) betont, dass funktionelle Verbesserungen zu Beginn der Erkrankung im postakuten Zustand erzielt werden können. Im späteren Verlauf werden funktionelle Verbesserungen immer schwieriger erreicht und der Schwerpunkt sollte daher auf die Krankheitsbewältigung gelegt werden. $\mathrm{Zu}$ Unterstreichung werden Beispiele aus dem Bereich der Aphasierehabilitation und aus Tiermodellen genannt. Dies ist auch aus berufspolitischer Perspektive höchst relevant, da der Stellenschlüssel in der neurologischen Frührehabilitation es häufig nicht ermöglicht, allen Betroffenen eine schnelle und intensive Therapie zu gewährleisten.

Sehr praxisrelevant ist zudem der Hinweis auf eine eventuelle Überforderung der Betroffenen im akuten Stadium. Wenn im akuten Stadium überhaupt Therapie möglich ist, dann bevorzugt am Krankenbett mit Fokus auf passiven Bottom-up-Methoden als Vorbereitung für später folgende Top-down-Methoden.

\section{Literatur}

Anderson, S.W. \& Tranel, D. (1989). Awareness of disease states following cerebral infarction, dementia, and head trauma: Standardized assessment. Clinical Neuropsychologist, 3, 327339. https://doi.org/10.1080/13854048908401482

Azouvi, P., Jacquin-Courtois, S. \& Luauté, J. (2017). Rehabilitation of unilateral neglect: Evidence-based medicine. Annals of Physical and Rehabilitation Medicine, 60, 191-197. https://doi. org/10.1016/j.rehab.2016.10.006

Bishop, M., Kayes, N. \& McPherson, K. (2021). Understanding the therapeutic alliance in stroke rehabilitation. Disability and Rehabilitation, 43,1074-1083. https://doi.org/10.1080/09638288. 2019.1651909

Hartman-Maeir, A., Soroker, N., Ring, H. \& Katz, N. (2002). Awareness of deficits in stroke rehabilitation. Journal of Rehabilitation Medicine, 34, 158-164. https://doi.org/10.1080/165019702 13236 
Hildebrandt, H. (2021). Zehn Argumente für dialogische Interaktion und kognitive Modelle als Grundlage neuropsychologischer Rehabilitation. Zeitschrift für Neuropsychologie, 32 (4), 229-242.

Leung, D.P.K. \& Liu, K.P.Y. (2011). Review of self-awareness and its clinical application in stroke rehabilitation. International Journal of Rehabilitation Research, 34, 187-195. https://doi. org/10.1097/MRR.0b013e3283487f31

Peiris, C.L., Taylor, N. F. \& Shields, N. (2012). Patients value patienttherapist interactions more than the amount or content of therapy during inpatient rehabilitation: A qualitative study. Journal of Physiotherapy, 58, 261-268. https://doi.org/10.1016/ S1836-9553(12)70128-5

Turgut, N., Miranda, M., Kastrup, A., Eling, P. \& Hildebrandt, H. (2018). TDCS combined with optokinetic drift reduces egocentric neglect in severely impaired post-acute patients. Neuropsychological Rehabilitation, 28, 515-526. https://doi.org/10.10 80/09602011.2016.1202120

\section{Historie}

Eingereicht: 14. September 2021

Akzeptiert: 16. September 2021

\section{Interessenkonflikt}

Die Autorin hat keine Interessenkonflikte.

\section{Dr. rer. nat Nergiz Turgut}

Institute of Neurosciences

Universidad San Francisco de Quito

Campus Cumbayá, Diego de Robles s/n

170901 Quito

Ecuador

nturgut@usfa.edu.ec 